FROM THE NATIONAL CENTER FOR HEALTH STATISTICS

\title{
Difference in Life Expectancy Between Females and Males at Birth and at Age 65 Years - National Vital Statistics System, United States, $1990,2000,2010$, and 2014
}

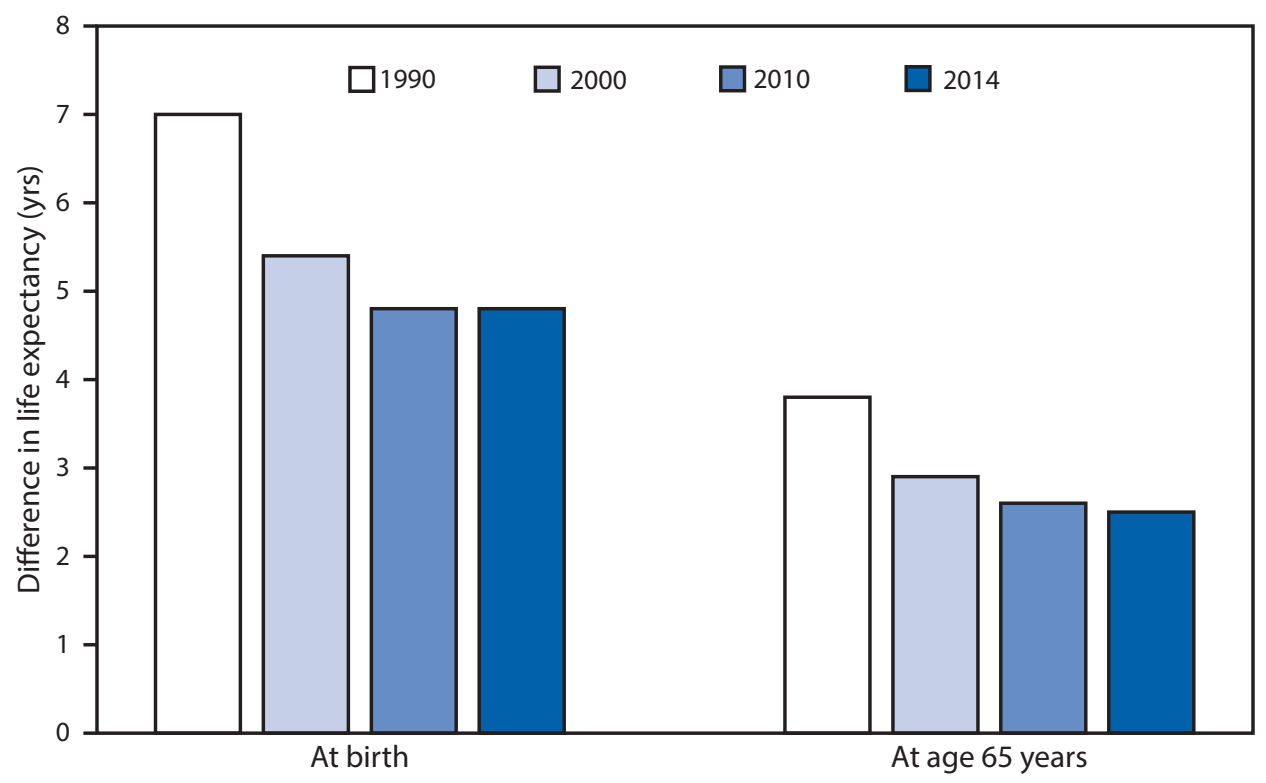

Females born in 2014 can expect to live 4.8 years longer than males born in the same year. This difference in life expectancy between females and males has not changed since 2010, but decreased from 5.4 years in 2000 and 7.0 years in 1990. The difference in life expectancy between females and males who were aged 65 years in 2014 was 2.5 years, a decrease from 2.6 years in 2010 , 2.9 years in 2000, and 3.8 years in 1990 .

Source: CDC. National Vital Statistics System. http://www.cdc.gov/nchs/nvss.htm.

Reported by: Yelena Gorina, MPH, MS, yag9@cdc.gov, 301-458-4241. 